
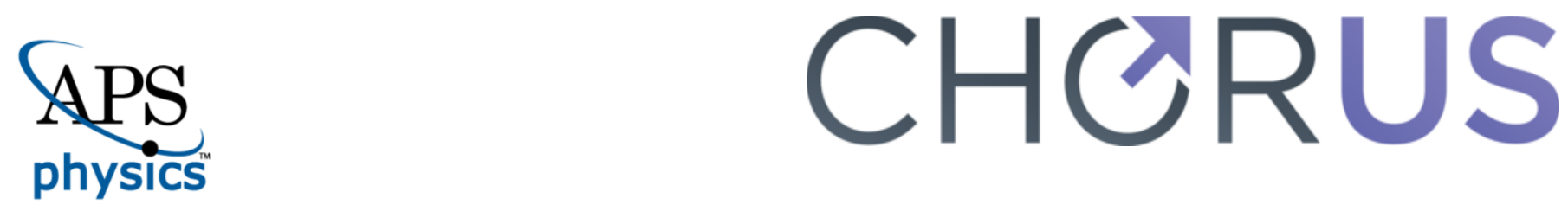

This is the accepted manuscript made available via CHORUS. The article has been published as:

\title{
Critical properties of the measurement-induced transition in random quantum circuits
}

Aidan Zabalo, Michael J. Gullans, Justin H. Wilson, Sarang Gopalakrishnan, David A. Huse, and J. H. Pixley

Phys. Rev. B 101, 060301 - Published 20 February 2020

DOI: 10.1103/PhysRevB.101.060301 


\title{
Critical properties of the measurement-induced transition in random quantum circuits
}

\author{
Aidan Zabalo, ${ }^{1}$ Michael J. Gullans, ${ }^{2}$ Justin H. Wilson, ${ }^{1}$ Sarang Gopalakrishnan, ${ }^{3}$ David A. Huse,,${ }^{2,}$ and J. H. Pixley ${ }^{1}$ \\ ${ }^{1}$ Department of Physics and Astronomy, Center for Materials Theory, Rutgers University, Piscataway, NJ 08854 USA \\ ${ }^{2}$ Physics Department, Princeton University, Princeton, New Jersey 08544, USA \\ ${ }^{3}$ Department of Engineering Science and Physics, CUNY College of Staten Island, \\ Staten Island, New York 10314, USA and Initiative for the Theoretical Sciences, \\ CUNY Graduate Center, New York, New York 10016 USA \\ ${ }^{4}$ Institute for Advanced Study, Princeton, New Jersey 08540, USA
}

(Dated: February 4, 2020)

\begin{abstract}
We numerically study the measurement-driven quantum phase transition of Haar-random quantum circuits in $1+1$ dimensions. By analyzing the tripartite mutual information we are able to make a precise estimate of the critical measurement rate $p_{c}=0.17(1)$. We extract estimates for the associated bulk critical exponents that are consistent with the values for percolation, as well as those for stabilizer circuits, but differ from previous estimates for the Haar-random case. Our estimates of the surface order parameter exponent appear different from that for stabilizer circuits or percolation, but we cannot definitively rule out the scenario where all exponents in the three cases match. Moreover, in the Haar case the prefactor for the entanglement entropies $S_{n}$ depends strongly on the Rényi index $n$; for stabilizer circuits and percolation this dependence is absent. Results on stabilizer circuits are used to guide our study and identify measures with weak finite-size effects. We discuss how our numerical estimates constrain theories of the transition.
\end{abstract}

Characterizing phase transitions in the dynamics of nonequilibrium quantum systems is a key open question in quantum statistical physics. So far, nonequilibrium phase transitions have been studied primarily for isolated quantum systems $[1,2]$ and for steady states of dissipative systems $[3,4]$. One much-studied case is the many-body localization transition [2], which can be seen either (i) as a dynamical transition at which thermalization slows down and stops as a parameter (e.g., the disorder strength in a spin chain) is tuned or (ii) as an entanglement transition at which the many-body eigenstates of the system change from volume-law to area-law entangled. Recently, a different type of entanglement transition was discovered [5-7] in the steady-state entanglement of the states produced by individual quantum trajectories [8-11] of a repeatedly-measured quantum many-body system. As the system is measured at an increasing rate, this single-trajectory entanglement goes from volume-law to area-law (see Fig. 1(a)) [6, 7, 12-18].

A measurement-driven transition is expected for quantum chaotic dynamics whether temporally random $[6,7]$ or Hamiltonian [19]. Current studies have focused on quantum circuits acting on an array of qudits (of local Hilbert space dimension $q$ ); these are believed to be generic models of chaotic quantum dynamics [20-26]. In specific cases, analytic results (or large-scale simulations) exist. For the Hartley entropy (i.e., rank of the reduced density matrix) and in the $q \rightarrow \infty$ limit, mappings to percolation have been found $[5,6,14,17]$. For stabilizer circuits, efficient classical simulations [7, 13, 16, 27] have been implemented in one-dimensional systems with $q=2$. All three methods agree (within numerical precision for the stabilizer circuits) on the order-parameter and correlation-length critical exponents (respectively $\eta$ and $\nu$ ) at the transition; all of them, likewise, pre- dict that the steady-state Rényi entanglement entropies, $S_{n}^{(A)}=(1-n)^{-1} \log _{2} \operatorname{Tr} \rho_{A}^{n}$ [where $A$ denotes a contiguous subsystem of length $L$ in a one-dimensional system, and $\rho_{A}$ its reduced density matrix] should scale as $S_{n} \sim \alpha_{n} \ln L$. For stabilizer circuits and in the large- $q$ limit, $\alpha$ is independent of $n$. However, the value of $\alpha$ seems to be different in each of these solvable cases, suggesting that in some respects these are distinct critical phenomena.

The present work analyzes the physically relevant, but analytically intractable, limit of Haar-random circuits with $q=2$. Some numerical results exist for this case $[6,14,16]$ but are inconclusive because the values of the critical exponents are sensitive to the estimate for the critical point and choice of scaling ansatz. We circumvent these issues by studying the tripartite mutual information (TMI), which is found to have minimal finite-size drifts and allows us to reliably locate the critical point with minimal scaling assumptions on small system sizes. The TMI is finite at the critical point, vanishes in the area-law phase, and diverges in the volume-law phase; thus, curves for different sizes cross at the critical point, allowing one to locate it reliably [13, 28, 29]. Having located the critical point, we estimate critical exponents; the correlation length exponent $\nu$ and the bulk anomalous dimension $\eta$ [27] (described below) for the Haar case are close to or equal to those for percolation. The surface critical exponent, however, appears to differ from both stabilizer circuits and percolation, suggesting that the Haar model lies in a separate universality class [30]. The Rényi entropies $S_{n}$ (for $n \geq 1$ ) appear to be logarithmic at the critical point, but with a strongly $n$-dependent prefactor: the entanglement spectrum has a nontrivial critical structure. To guide our study of Haar-random circuits, we analyze small stabilizer circuits using the 
(a)

(a) Volume Law

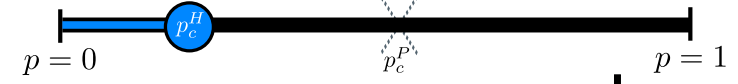

(b)

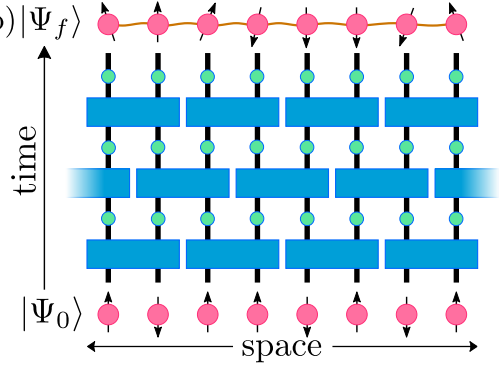

(c)

(d)

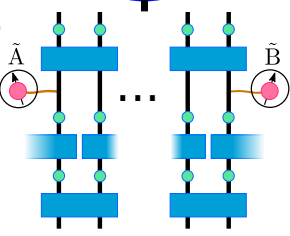

FIG. 1. (a) Phase diagram with $p_{c}^{H}$ and $p_{c}^{P}$ marking the separation between volume law and area law entanglement in the Rényi entropies $n \geq 1$ and $n=0$, respectively. (b) Depiction of the model: Blue rectangles represent two-site entangling gates and the green circles denote local projective measurements that are performed with a probability $p$. (c) The geometry used to compute the TMI, partitioning the system with periodic boundary conditions into four equal-length segments. (d) The set-up to probe the order parameter correlation function through entangling the local system qubits at time $t=t_{0}=2 L$ with two ancilla qubits seperated by a distance $r-r^{\prime}$ and computing their mutual information at later times.

same methods: our results for small sizes reliably predict the exponents found from much larger sizes, showing that our observables have weak finite-size effects in stabilizer circuits, and thus seem likely to also be well-behaved for Haar-random circuits.

Models. - We focus on two different models of random circuits in a "brick-layer" geometry with local projective measurements, as shown in Fig. 1(b). We start from a trivial product state $\left|\Psi_{0}\right\rangle$ then time evolve in the presence of measurements. In the following, we consider two circuit models specified by the distribution of the gates. The local two-qubit gates $U_{i, i+1}$ (depicted as blue rectangles) are drawn from a Haar-random distribution for the Haar circuit model and for the stabilizer circuit model they are sampled uniformly from the Clifford group. We expect the behavior of the Haar model to capture the generic behavior of systems undergoing chaotic unitary dynamics interspersed with projective measurements. At each space-time "site" $(j)$ [shown as a green circle in Fig. 1(b)] with probability $p$ we make a measurement of the $z$-component of the spin $S_{j}^{z}$, project onto the measured value of $S^{z}$, and normalize the state. For the Haar simulation, we exactly time evolve the state, while for the Clifford gates we initialize the system in a stabilizer state and dynamically update a generating set for the stabilizer group of this state [31]. To reduce finite size effects, we use periodic boundary conditions for a system size $L$, unless otherwise specified. We define one time step as one layer of gates and one layer of measurements.
Locating the critical point.- Natural diagnostics of the transition are the bipartite Rényi entropies $S_{n}$, which saturate to a steady state on times $t \sim L$. However, these entropies diverge logarithmically with $L$ at the critical point. Locating the critical point via $S_{n}$ requires one to account for the logarithm, which makes the finite-size scaling behavior less constrained. To circumvent this issue, we focus on the TMI between regions $A, B$, and $C$ as depicted in Fig. 1(c)

$$
\begin{aligned}
& \mathcal{I}_{3, n}(A, B, C) \equiv S_{n}(A)+S_{n}(B)+S_{n}(C)-S_{n}(A \cup B) \\
& -S_{n}(A \cup C)-S_{n}(B \cup C)+S_{n}(A \cup B \cup C) .
\end{aligned}
$$

We run the circuit out to time $t=4 L$ so that the data is solely dependent on system size [32]. In the area law phase, $\mathcal{I}_{3}$ is asymptotically zero for large $L$ because all the contributions to it come from boundary terms, and the boundary terms cancel out exactly in Eq. (1). In the volume law phase, it is negative and proportional to $L$, as the "bulk" contributions from regions $A, B$, and $C$ get subtracted out twice. We find that $\mathcal{I}_{3}$ is finite and negative at the critical point. Within the minimal cut picture (which does not strictly apply to $n>0$, but appears to qualitatively capture some aspects of the transition) one can understand the behavior of $\mathcal{I}_{3}$ analytically [32]. We remark that within the minimal cut picture, the mutual information $\mathcal{I}_{2, n}(A, C) \equiv S_{n}(A)+S_{n}(C)-S_{n}(A \cup C)$ should also be a constant at the critical point. Empirically, however, $\mathcal{I}_{2}$ has large finite-size drifts at small sizes [32].

We now turn to our numerical results on general $\mathcal{I}_{3, n}$. We find that $\mathcal{I}_{3, n}$ is an $\mathrm{O}(1)$ system size independent number at criticality for all values of $n$ we have considered [32]. Thus, consistent with the minimal-cut argument as well as previous results on stabilizer circuits [13]. Our results for $\mathcal{I}_{3, n=1}$ are shown in Fig. 2(a) for Clifford gates and (b) for Haar gates at late times $(t=4 L)$ and similar system sizes. The TMI is negative for all $p$ and the data for different system sizes has a clear crossing for system sizes $L=16,20,24$. For stabilizer circuits this crossing yields an estimate of $p_{c}^{C}=0.154(4)$, close to the critical value obtained up to sizes $L=512$. For the Haar case, we estimate $p_{c}^{H}=0.168(5)$. The value of $\mathcal{I}_{3, n}\left(p_{c}\right)$ is $L$-independent; for Haar gates we find $\mathcal{I}_{3, n=1}\left(p_{c}^{H}\right) \approx-0.66(8)$ and for stabilizer circuits $\mathcal{I}_{3}^{C}\left(p_{c}^{C}\right) \approx-0.56(9)$. The location of the crossings of the TMI for $n>1$ [32] give estimates of $p_{c}$ that agree to within error bars with the result for $n=1$.

Correlation-length exponent.-For $\mathcal{I}_{3, n=1}$ at late times $(t=4 L)$, we apply the scaling hypothesis

$$
\mathcal{I}_{3, n=1}(p, L) \sim f\left(L^{1 / \nu}\left(p-p_{c}\right)\right)
$$

where $f(x)$ is a scaling function and $\nu$ is the correlation length exponent. As shown in Fig. 2(c) and (d) we find excellent data collapse that yields $\nu^{C}=1.24(7)$ and $\nu^{H}=$ $1.2(2)$, respectively. Our results for stabilizer circuits on 

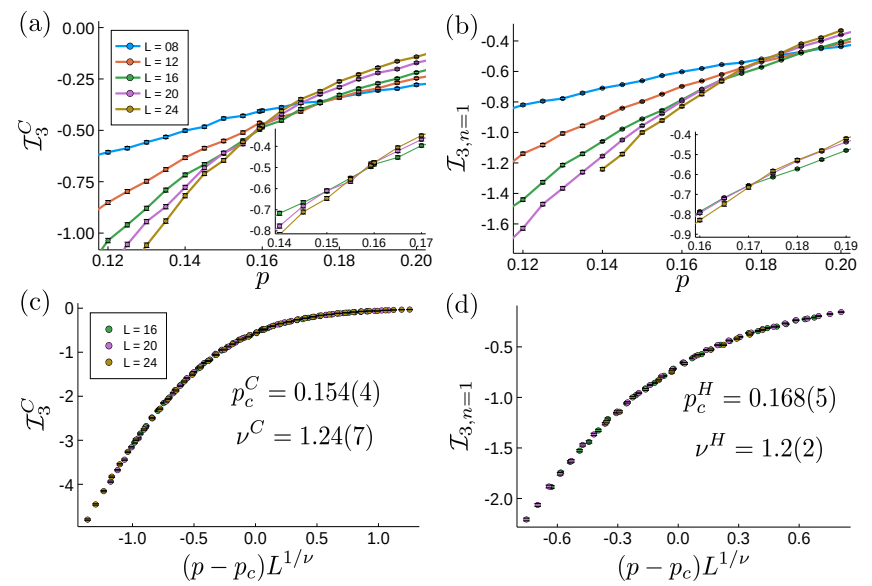

FIG. 2. Tripartite mutual information (TMI) near the transition. TMI near the transition for a circuit with (a) Clifford and (b) Haar gates. Scaling collapse of the data for (c) Clifford and (d) Haar gates.

small sizes agree with results on much larger system sizes up to $L=512$. We also obtain $\nu^{H}$ for various other Rényi indices and find that $\nu^{H}$ varies across $1.2(2)$ to $1.4(1)$ from $n=0.7$ to $\infty$ (see Table I and [32]), suggesting that $\nu^{H}$ is constant for all $n \geq 1$.

For $n>1$ one can see that $p_{c}$ and $\nu$ are $n$-independent. All $S_{n}(n>1)$ are upper- and lower-bounded by $S_{\infty}$ : $S_{\infty} \leq S_{n} \leq n /(n-1) S_{\infty}$. Thus if any $S_{n}$ with $n>1$ scales as a volume law, so must the others. Assuming single-parameter scaling as in Eq. (2), $\nu^{H}$ is also independent of $n$ : In the volume-law phase, by assumption, $S_{n}(L) \sim f_{n}\left(L / \xi_{n}\right)$ where $f_{n}(x) \sim \alpha_{n} \ln x$ for $x \ll 1$ and $f_{n}(x) \sim \alpha_{n}^{\prime} x$ for $x \gg 1$ as well as $\xi_{n} \sim\left|p-p_{c}\right|^{-\nu_{n}^{H}}$. Then at very large length-scales, $S_{n} / S_{\infty} \sim \alpha_{n}^{\prime} / \alpha_{\infty}^{\prime}\left(\xi_{\infty} / \xi_{n}\right)$. This quantity cannot get parametrically large without violating the bounds on $S_{n}$, so $\xi_{\infty} / \xi_{n}$ must approach a constant, so $\nu^{H}$ is $n$-independent for $n>1$. For $n=1$ these bounds do not apply. One can argue that $p_{c}$ remains $n$-independent for $0<n<1$ assuming the entanglement transition coincides with the purification transition for an ancilla qubit (see below). For $p>p_{c}$, the ancilla purifies exponentially at a rate $t \sim L$; its smaller Schmidt coefficient decreases exponentially at this rate, so all its nonzero Rényi entropies vanish on timescales $\sim L$, yielding the same $p_{c}$ for all $n>0$.

The values of $\nu^{H}$ and $\nu^{C}$ are similar; indeed, within our numerical uncertainty both are consistent with the percolation exponent $\nu^{P}=4 / 3$. For a more thorough comparison between stabilizer and Haar circuits we now turn to order-parameter correlations.

Order parameter.-A local bulk order parameter for the volume-law phase can be defined as follows [27]. We run the circuit out to a steady state, then place one of the system spins into a Bell state with a reference qubit (an ancilla) $R$ at time $t=t_{0}$. We continue running the unitary-projective dynamics on the system. At $t_{0}$ the state of the system and $R$ is $\left|\psi_{0}\right\rangle=\frac{1}{\sqrt{2}}|A \uparrow\rangle-|B \downarrow\rangle$, where $|A\rangle,|B\rangle$ are orthogonal states of the system that are locally distinguishable at $t_{0}$. The order parameter is then $S_{1}\left(\rho_{R}\right)$, where $\rho_{R}$ is the density matrix of $R$ at a time $\left(t-t_{0}\right) \gg L$. This behaves differently in the two phases: In the area-law phase, measurements collapse the local state of the system that is coupled to $R$, thus disentangling $R$ and driving the order parameter to zero. In the volume-law phase the states $A$ and $B$ become indistinguishable under local measurements, and thus remain linearly independent under the dynamics, so the reference qubit stays entangled with the system and the order parameter remains nonzero. Analogous surface order parameters can be defined by entangling $R$ with the initial state at $t_{0}=0$, or by using open boundary conditions and entangling it with an end spin. The estimate of $p_{c}$ obtained from order parameter dynamics agrees with that obtained from the TMI [32].

At $p_{c}$ the bulk order parameter decays very slowly; to get a cleaner numerical signal we study its two-point correlation function, which we access by introducing two ancilla qubits, $\tilde{A}$ and $\tilde{B}$, and entangling them with circuit qubits at spacetime points $\left(r, t_{0}\right)$ and $\left(r^{\prime}, t_{0}\right)$ [see Fig. 1(d)]. We define the connected order-parameter correlation as the mutual information between these ancillas. We fix $r-r^{\prime}$ and determine the time dependence of the mutual information between $\tilde{A}$ and $\tilde{B}$, denoted $C\left(t-t_{0}\right)$. We consider two separate geometries: (i) periodic boundary conditions, with ancillas connected to antipodal sites $\left(r-r^{\prime}=L / 2\right)$, and (ii) open boundary conditions, with ancillas connected to spins at each edge $\left(r-r^{\prime}=L-1\right)$. In both cases we start from a product state and run the circuit out to a time $t_{0}=2 L$, introduce the ancilla qubits, maximally entangle them, and track their mutual information. Through a conformal transformation, the scaling dimension of case (ii) can be related to the surface exponent $\eta_{\|}[32]$.

Our results for $C\left(t-t_{0}\right)$ are shown in Fig. 3. In both the volume law phase, $p<p_{c}$, and the area law phase, $p>p_{c}, C\left(t-t_{0}\right) \sim \exp (-L / \xi)$ for $L \gg \xi$, where $\xi$ here is a finite correlation length away from $p_{c}$. At criticality, we numerically estimate the dynamic exponent $z=1.06(4)$ [32], which is consistent with previous work that finds $z=1[5-7,16]$. Using this, the single-parameter scaling hypothesis implies the form

$$
C\left(t-t_{0}, L\right) \sim L^{-\eta} g\left(\left(t-t_{0}\right) / L\right)
$$

where $\eta$ and $g(x)$ depend on the boundary conditions used. As demonstrated in Fig. 3, we find excellent data collapse for system sizes $L=12,16,20$ for both Haar and Clifford gates, and a summary of the exponents are given in Table I. For periodic boundary conditions we find $\eta^{C}=0.22(1)$ for Clifford gates and $\eta^{H}=0.19(1)$ for Haar gates. Again, this result for Clifford gates agrees 

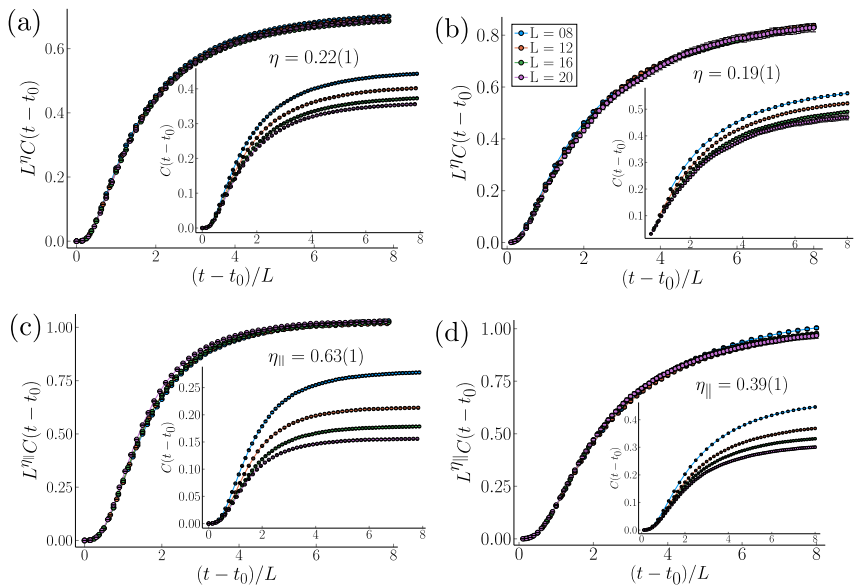

FIG. 3. Scaling collapse of mutual information between two ancilla qubits (inset: unscaled data). Mutual information between ancilla entangled at $r-r^{\prime}=L / 2$ for (a) Clifford and (b) Haar gates with periodic boundary conditions. Mutual information between ancilla entangled at $r=1, r^{\prime}=L$ for (c) Clifford and (d) Haar gates with open boundary conditions.

with a similar analysis at much larger $L$ [27]. These bulk exponents are both within uncertainties of the percolation value $\eta^{P}=5 / 24$. To estimate the surface critical exponent we consider open boundary conditions and find $\eta_{\|}^{H}=0.39(1)$ and $\eta_{\|}^{C}=0.63(1)$, the latter of which is consistent with results obtained in different geometries on sizes up to $L=1024$ [27] and close to the percolation value of $2 / 3$. In stabilizer circuits, $\eta_{\|}$extracted from the geometry used in Fig. 3(c) has the smallest finite size effects, and the large discrepancy between these values suggests that the Haar and stabilizer circuits are in different universality classes [30]. We have also checked whether this discrepancy persists in other geometries that have larger finite size effects for the stabilizer circuits [32]. The statistical error in the collapse for these other quantities is not high, but certain exact relations based on the scaling hypothesis are not satisfied, so there are potentially large systematic uncertainties in our estimate of $\eta_{\|}$. Although our results suggest a different exponent, we cannot rule out a scenario in which the surface exponent is also consistent with percolation.

Finally, the order parameter dynamics for Haar and stabilizer circuits is qualitatively different. In stabilizer circuits, the ancilla jumps from fully mixed to fully pure in a single timestep; by contrast, in the Haar case, individual realizations purify gradually [32].

Rényi Entropies.-We now turn to the behavior of the Rényi entropies, which provides a clear distinction between Haar and stabilizer circuits. For stabilizer circuits at criticality we find $S_{n}\left(p_{c}, L\right) \sim \alpha_{C} \ln L$ for all $n$ with $\alpha_{C}=1.61(3)$ on system sizes up to $L=24$, which agrees well with a similar fit out to much larger $L$ [yielding 1.63(3)]. In contrast, our data for Haar random circuits

\begin{tabular}{ccccccc}
\hline \hline$n$ & 1 & 2 & 5 & $\infty$ & $\mathrm{C}$ & $\mathrm{P}$ \\
\hline$p_{c}$ & $0.168(5)$ & $0.162(3)$ & $0.168(4)$ & $0.170(4)$ & $0.154(4)$ & 0.5 \\
\hline$\nu$ & $1.2(2)$ & $1.3(1)$ & $1.4(1)$ & $1.4(1)$ & $1.24(7)$ & 1.33 \\
\hline$\eta$ & $0.19(1)$ & $0.25(1)$ & $0.26(1)$ & $0.26(1)$ & $0.22(1)$ & 0.21 \\
\hline$\eta_{\|}$ & $0.39(1)$ & $0.49(1)$ & $0.49(2)$ & $0.49(2)$ & $0.63(1)$ & 0.67 \\
\hline$\eta_{\perp}$ & $0.23(2)$ & $0.31(2)$ & $0.34(1)$ & $0.34(1)$ & $0.43(2)$ & 0.44 \\
\hline$\alpha(n)$ & $1.7(2)$ & $1.2(2)$ & $0.9(1)$ & $0.7(1)$ & $1.61(3)$ & 0.55 \\
\hline \hline
\end{tabular}

TABLE I. Table listing critical properties as a function of Rényi index $n$. The column $C$ corresponds to the $n$ independent results for the stabilizer circuit at small $L$ and $\mathrm{P}$ to the exact results from percolation provided to two digits of accuracy [33].
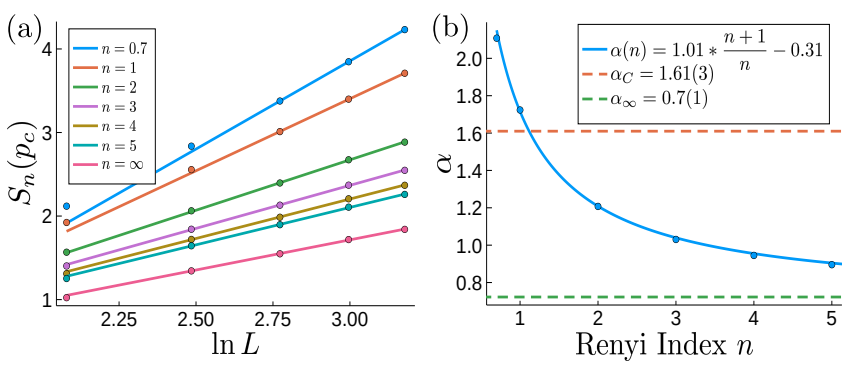

FIG. 4. Properties of the Rényi entropies at criticality. (a) The Rényi entropies show a $\ln L$ dependence near the critical point estimated by $\mathcal{I}_{3, n}$ for $n=0.7,1,2,3,4,5, \infty$, with fits shown as solid lines. (b) The coefficient of the $\ln L$ term has a strong Rényi index dependence that is well described by a functional form $a(1+1 / n)+b$.

has a clear dependence on $n$, as shown in Fig. 4: we find

$$
S_{n}\left(p_{c}, L\right) \sim \alpha(n) \ln L, \alpha(n)=0.7(1)+1.0(1) / n .
$$

This fit is consistent with our direct estimate of $\alpha(n=$ $\infty) \approx 0.7$. Interestingly, $\alpha(\infty)$ is close to the percolation value $(=\sqrt{3} / \pi \approx 0.55$ for periodic boundary conditions [34]), while $\alpha(1)$ is not far from the stabilizer value.

Discussion. - The critical properties obtained here are summarized in Table I. With our improved estimate of $p_{c}$, any differences in the bulk critical exponents between percolation and the Haar and stabilizer circuit transitions are within our uncertainties. Haar and stabilizer circuits apparently differ in the surface critical exponent $\eta_{\|}$and clearly differ in the coefficients $\alpha(n)$ of the log-divergence in the Rényi entropies at criticality. Constraints imposed by conformal invariance imply that a different value of the surface critical exponent from percolation would imply that the Haar-random model is in a separate universality class [30]. The Rényi-dependence fits to a form $b+a(1+1 / n)$, which is reminiscent of the scaling form for unitary conformal field theories, $a(1+1 / n)[35]$; however, in the present case one needs an offset to fit the data, so the critical wavefunctions at the measurement-induced transition differ from critical ground states. We stress 
that these results are beyond any current analytic estimates, and come from being in the fully quantum regime: the Rényi-dependence is trivial in all the solvable limits.

The overall picture that emerges from our results is that the distinctions between the three known classes (percolation, stabilizer circuits, Haar-random circuits) of measurement-induced criticality are rather subtle: The correlation length and bulk order-parameter exponents are consistent in all three cases to within our present error estimates. However, the entanglement entropies at the critical points are clearly different, and the surfaceorder parameter exponents appear to differ. Understanding why these superficially distinct critical phenomena look so similar is an important challenge for future work.

We thank Ehud Altman, Soonwon Choi, Vedika Khemani, Andreas Ludwig, Adam Nahum, Xiaoliang Qi, Jonathan Ruhman, Brian Skinner, and Romain Vasseur for helpful discussions. A. Z. is partially supported through a Fellowship from the Rutgers Discovery Informatics Institute. M.G. and D.H. are supported in part by the DARPA DRINQS program. S.G. acknowledges support from NSF Grant No. DMR-1653271. D.H. is also supported in part by a Simons Fellowship. J.H.P. is partially supported by Grant No. 2018058 from the United States-Israel Binational Science Foundation (BSF), Jerusalem, Israel. S.G. and J.H.P. initiated this work at the Kavli Institute for Theoretical Physics, which is supported by NSF Grant No. PHY-1748958 and J.H.W, S.G. and J.H.P. performed part of this work at the Aspen Center for Physics, which is supported by NSF Grant No. PHY- 1607611. The authors acknowledge the Beowulf cluster at the Department of Physics and Astronomy of Rutgers University, the Office of Advanced Research Computing (OARC) at Rutgers, The State University of New Jersey (http://oarc.rutgers.edu) for providing access to the Amarel cluster, the Rutgers Discovery Informatics Institute for providing access to the Caliburn [36] cluster supported by Rutgers and the State of New Jersey, and associated research computing resources that have contributed to the results reported here.

[1] A. Polkovnikov, K. Sengupta, A. Silva, and M. Vengalattore, Rev. Mod. Phys. 83, 863 (2011).

[2] R. Nandkishore and D. A. Huse, Annu. Rev. Condens. Matter Phys. 6, 15 (2015).

[3] H.-P. Breuer and F. Petruccione, The Theory of Open Quantum Systems (Oxford University Press, 2002).

[4] L. M. Sieberer, M. Buchhold, and S. Diehl, Rep. Prog. Phys. 79, 096001 (2016).

[5] R. Vasseur, A. C. Potter, Y.-Z. You, and A. W. W. Ludwig, Phys. Rev. B 100, 134203 (2019).

[6] B. Skinner, J. Ruhman, and A. Nahum, Phys. Rev. X 9, 031009 (2019).
[7] Y. Li, X. Chen, and M. P. A. Fisher, Phys. Rev. B 98, 205136 (2018).

[8] P. Zoller, M. Marte, and D. F. Walls, Phys. Rev. A 35, 198 (1987).

[9] K. Mølmer, Y. Castin, and J. Dalibard, JOSA B 10, 524 (1993).

[10] H. J. Carmichael, An Open Systems Approach to Quantum Optics (Springer-Verlag, Berlin, 1993).

[11] M. B. Plenio and P. L. Knight, Rev. Mod. Phys. 70, 101 (1998).

[12] S. Choi, Y. Bao, X.-L. Qi, and E. Altman, (2019), arXiv:1903.05124.

[13] M. J. Gullans and D. A. Huse, (2019), arXiv:1905.05195.

[14] Y. Bao, S. Choi, and E. Altman, (2019), arXiv:1908.04305.

[15] M. Szyniszewski, A. Romito, and H. Schomerus, Phys. Rev. B 100, 064204 (2019).

[16] Y. Li, X. Chen, and M. P. A. Fisher, Phys. Rev. B 100, 134306 (2019).

[17] C.-M. Jian, Y.-Z. You, R. Vasseur, and A. W. W. Ludwig, "Measurement-induced criticality in random quantum circuits," (2019), arXiv:1908.08051.

[18] A. Chan, R. M. Nandkishore, M. Pretko, and G. Smith, Phys. Rev. B 99, 224307 (2019).

[19] Q. Tang and W. Zhu, (2019), arXiv:1908.11253.

[20] A. Nahum, J. Ruhman, S. Vijay, and J. Haah, Phys. Rev. X 7, 031016 (2017).

[21] T. Rakovszky, F. Pollmann, and C. W. von Keyserlingk, Phys. Rev. X 8, 031058 (2018).

[22] C. W. von Keyserlingk, T. Rakovszky, F. Pollmann, and S. L. Sondhi, Phys. Rev. X 8, 021013 (2018).

[23] V. Khemani, A. Vishwanath, and D. A. Huse, Phys. Rev. X 8, 031057 (2018).

[24] T. Rakovszky, F. Pollmann, and C. W. von Keyserlingk, Phys. Rev. X 8, 031058 (2018).

[25] A. Chan, A. De Luca, and J. T. Chalker, Phys. Rev. X 8, 041019 (2018).

[26] P.-Y. Chang, X. Chen, S. Gopalakrishnan, and J. H. Pixley, Phys. Rev. Lett. 123, 190602 (2019).

[27] M. J. Gullans and D. A. Huse, (2019), arXiv:1910.00020.

[28] A. Kitaev and J. Preskill, Phys. Rev. Lett. 96, 110404 (2006).

[29] M. Levin and X.-G. Wen, Phys. Rev. Lett. 96, 110405 (2006).

[30] J. L. Cardy, Nucl. Phys. B 240, 514 (1984).

[31] S. Aaronson and D. Gottesman, Phys. Rev. A 70, 052328 (2004).

[32] See online supplemental material for details. See also Ref. [37, 38].

[33] D. Stauffer and A. Aharony, Introduction to Percolation Theory (Taylor and Francis, London/Philadelphia, 2003).

[34] J. Cardy, Phys. Rev. Lett. 84, 3507 (2000).

[35] P. Calabrese and A. Lefevre, Phys. Rev. A 78, 032329 (2008).

[36] J. J. Villalobos, M. Parashar, I. Rodero, and M. Brennan-Tonetta, "High performance computing at the rutgers discovery informatics institute," (2018).

[37] N. Kawashima and N. Ito, J. Phys. Soc. Jpn. 62, 435 (1993).

[38] M. Kardar and Y.-C. Zhang, Phys. Rev. Lett. 58, 2087 (1987). 